\title{
Disposable platform provides visual and color-based point-of-care anemia self-testing
}

\author{
Erika A. Tyburski, ${ }^{1,2,3,4,5}$ Scott E. Gillespie, ${ }^{3}$ William A. Stoy, ${ }^{1,4}$ Robert G. Mannino, ${ }^{1,2,3,4,5}$ Alexander J. Weiss, ${ }^{1}$ Alexa F. Siu, ${ }^{1}$ \\ Rayford H. Bulloch, ${ }^{6}$ Karthik Thota, ${ }^{1}$ Anyela Cardenas, ${ }^{7}$ Wilena Session, ${ }^{5}$ Hanna J. Khoury, ${ }^{5}$ Siobhán O'Connor, ${ }^{8}$ Silvia T. Bunting, ${ }^{7}$ \\ Jeanne Boudreaux, ${ }^{2,3}$ Craig R. Forest, ${ }^{4,9}$ Manila Gaddh, ${ }^{5}$ Traci Leong, ${ }^{10}$ L. Andrew Lyon, ${ }^{4,6}$ and Wilbur A. Lam ${ }^{1,2,3,4,5}$ \\ 'Wallace H. Coulter Department of Biomedical Engineering, Georgia Institute of Technology and Emory University, Atlanta, Georgia, USA. Aflac Cancer Center and Blood Disorders Service of Children's \\ Healthcare of Atlanta, Atlanta, Georgia, USA. ${ }^{3}$ Department of Pediatrics, Emory University School of Medicine, Atlanta, Georgia, USA. ${ }^{4}$ Parker H. Petit Institute of Bioengineering and Bioscience, \\ Georgia Institute of Technology, Atlanta, Georgia, USA. ${ }^{5}$ Winship Cancer Institute, Emory University, Atlanta, Georgia, USA. ${ }^{6}$ School of Chemistry and Biochemistry, Ceorgia Institute of Technology, \\ Atlanta, Georgia, USA. 'Department of Pathology, Children's Healthcare of Atlanta, Atlanta, Georgia, USA. ${ }^{8}$ US Centers for Disease Control and Prevention, Atlanta, Georgia, USA. \\ ${ }^{9}$ George W. Woodruff School of Mechanical Engineering, Georgia Institute of Technology, Atlanta, Georgia, USA. ${ }^{10}$ Department of Biostatistics \& Bioinformatics, \\ Rollins School of Public Health, Emory University, Atlanta, Georgia, USA.
}

BACKGROUND. Anemia, or low blood hemoglobin (Hgb) levels, afflicts 2 billion people worldwide. Currently, Hgb levels are typically measured from blood samples using hematology analyzers, which are housed in hospitals, clinics, or commercial laboratories and require skilled technicians to operate. A reliable, inexpensive point-of-care (POC) Hgb test would enable cost-effective anemia screening and chronically anemic patients to self-monitor their disease. We present a rapid, standalone, and disposable POC anemia test that, via a single drop of blood, outputs color-based visual results that correlate with Hgb levels.

METHODS. We tested blood from 238 pediatric and adult patients with anemia of varying degrees and etiologies and compared hematology analyzer Hgb levels with POC Hgb levels, which were estimated via visual interpretation using a color scale and an optional smartphone app for automated analysis.

RESULTS. POC Hgb levels correlated with hematology analyzer Hgb levels ( $r=0.864$ and $r=0.856$ for visual interpretation and smartphone app, respectively), and both POC test methods yielded comparable sensitivity and specificity for detecting any anemia $(n=178)(<11 \mathrm{~g} / \mathrm{dl}$ ) (sensitivity: $90.2 \%$ and $91.1 \%$, specificity: $83.7 \%$ and $79.2 \%$, respectively) and severe anemia ( $n=10)(<7 \mathrm{~g} / \mathrm{dl}$ ) (sensitivity: $90.0 \%$ and $100 \%$, specificity: $94.6 \%$ and $93.9 \%$, respectively).

CONCLUSIONS. These results demonstrate the feasibility of this POC color-based diagnostic test for self-screening/selfmonitoring of anemia.

TRIAL REGISTRATION. Not applicable.

FUNDING. This work was funded by the FDA-funded Atlantic Pediatric Device Consortium, the Georgia Research Alliance, Children's Healthcare of Atlanta, the Georgia Center of Innovation for Manufacturing, and the InVenture Prize and Ideas to Serve competitions at the Georgia Institute of Technology.

\section{Introduction}

Anemia, defined by low hemoglobin ( $\mathrm{Hgb}$ ) concentrations in the blood, affects one-third of the world's population, approximately 2 billion people, and is especially prevalent in young children, women of childbearing age, and the elderly (1). Symptoms of anemia range from weakness, fatigue, and dizziness in milder cases to life-threat-

Conflict of interest: The authors have declared that no conflict of interest exists Role of funding source: The study was designed, conducted, analyzed, and reported entirely by the authors. The Georgia Research Alliance and FDA-funded Atlantic Pediatric Device Consortium provided funding support through phase I funding programs. Children's Healthcare of Atlanta, the Georgia Center of Innovation for Manufacturing, and the Georgia Tech InVenture Prize and Ideas to Serve competitions provided funding for test supplies and other clinical assessment resources.

Submitted: April 16, 2014; Accepted: July 24, 2014.

Reference information: J Clin Invest. 2014;124(10):4387-4394. doi:10.1172/JCI76666. ening cardiovascular collapse in more severe cases, and chronic anemia leads to permanent neurocognitive deficits in children $(2,3)$. The most common causes of anemia are nutritional deficiencies (e.g., iron deficiency), primary hematologic diseases (e.g., sickle cell disease, thalassemia, myelodysplastic syndromes), drugs (e.g., cancer chemotherapy), and other chronic conditions that indirectly cause anemia (e.g., chronic kidney disease, inflammatory/autoimmune disorders, HIV) (4-7). Even in developed nations, anemia remains a major public health issue, as an estimated 85 million people are at high risk for anemia and 5 million people are afflicted by anemia, respectively, in the US alone (8-11).

Currently, the gold standard for anemia diagnosis is a complete blood count (CBC) using a hematology analyzer, which measures the $\mathrm{Hgb}$ levels of a venous blood sample. As CBCs require a venous blood draw from a trained phlebotomist and hematology 

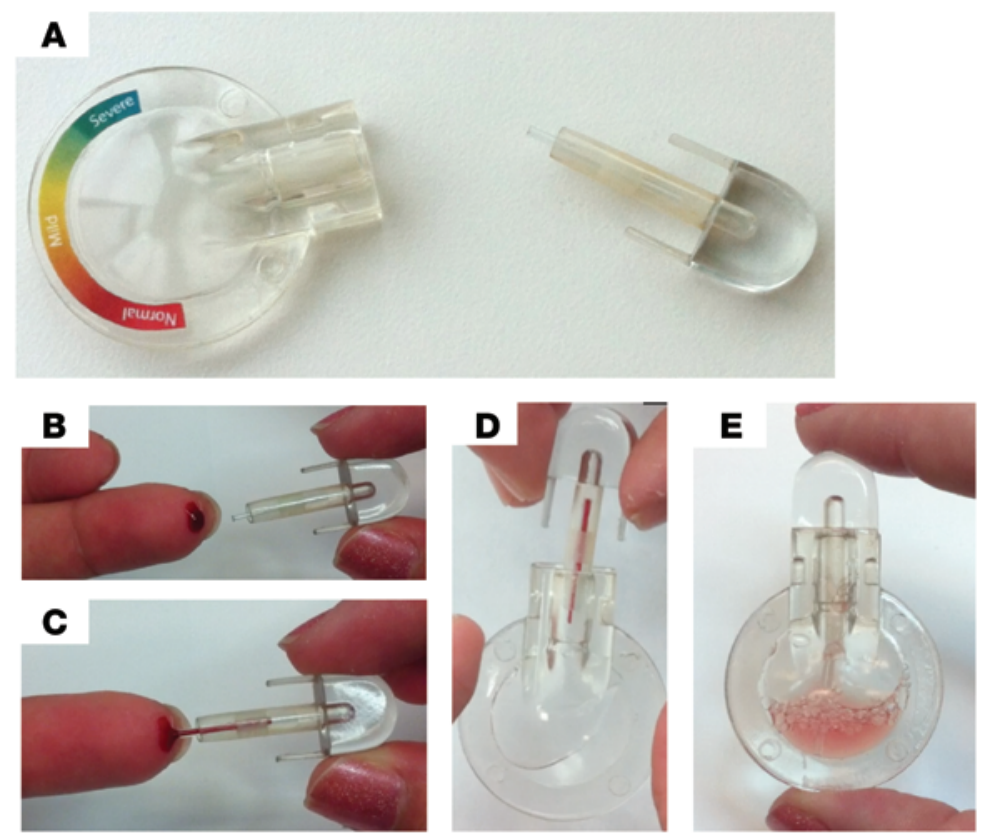

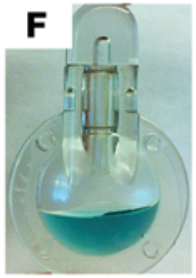

$7.2 \mathrm{~g} / \mathrm{dl}$

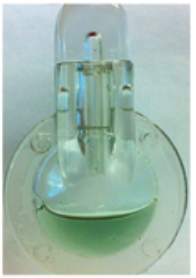

$9.1 \mathrm{~g} / \mathrm{dl}$

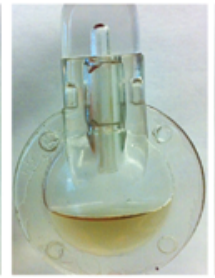

$10.5 \mathrm{~g} / \mathrm{dl}$

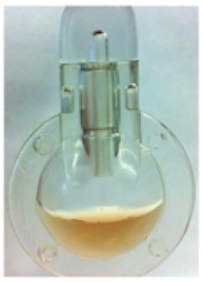

$12.0 \mathrm{~g} / \mathrm{dl}$



$14.1 \mathrm{~g} / \mathrm{dl}$

Hgb levels

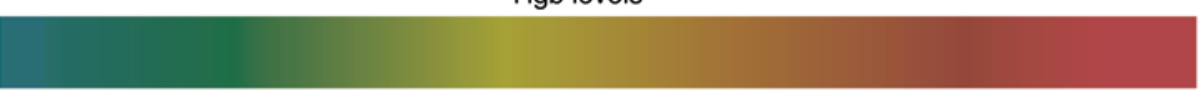

Figure 1. The POC color-based anemia test. (A) The device is comprised of 2 parts, a cap and a body. A 5- $\mu$ l sample tube for precise and accurate blood collection is embedded in the cap. The body contains prefilled reagent solution that changes color when in contact with blood. A color scale sticker on the body enables user interpretation of the degree of anemia, if any. (B-E) Steps for using the POC color-based anemia test. (B) Perform a finger stick. (C) Collect blood until sample tube (within the cap) is filled via capillary action. (D) Insert cap into body and lock in place. (E) Mix and wait for 60 seconds and interpret resultant solution color. (F) Ranging from blue to red, resultant solution colors correlate with different Hgb levels, as indicated.

analyzers require a skilled technician and electrical power to operate, these devices are currently housed in hospitals, clinics, and commercial laboratories, necessitating patients to travel outside of their home to be tested for anemia. With anemia's widespread prevalence, an inexpensive, disposable, stand-alone point-of-care (POC) anemia diagnostic that is simple enough for untrained people to use as a self-test will enable (a) patients with chronic anemia to self-monitor their disease, improve their quality of life, and potentially improve clinical outcomes and (b) the general public to self-screen for anemia in a cost-effective manner. While several POC anemia diagnostics are currently available, all are currently designed for use by healthcare professionals and none are designed for home use as a self-test (12-16).

To those ends, here we present a visual color-based POC anemia test. The assay is rapid (<60 s), simple to use, disposable, and inexpensive and is a stand-alone system that does not require electrical power, additional equipment, or significant training.

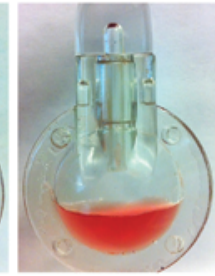

$16.3 \mathrm{~g} / \mathrm{dl}$
Therefore, the device is feasible as a home-based test for self-screening or self-monitoring of anemia. Each test requires a single $5 \mu$ drop of capillary blood obtained via finger stick and can be interpreted with the naked eye. A clinical assessment study was conducted using blood samples from patients with anemia of different degrees and etiologies comparing visual interpretation of our POC test results by untrained users with clinical CBC Hgb levels obtained simultaneously. In addition, we present results from an optional custom color analysis smartphone app that integrates with the POC anemia test for automated Hgb level measurements and potential data transmission.

\section{Results}

Our color-based POC anemia test differentiates among different degrees of anemia with a visual readout from a quick, inexpensive, disposable, and stand-alone device that does not require any additional equipment or electrical power (Figure 1 and STARD flowchart; the STARD flowchart can be found in the supplement; supplemental material available online with this article; doi:10.1172/ JCI76666DS1). The device consists of 2 parts, a cap and a body, with a color scale sticker attached to the latter (Figure 1A). The cap encases a sample tube for blood collection, and, after a finger stick is performed (Figure 1B), blood is collected via capillary action as it comes into contact with the sample tube (Figure 1C). Capillary action enables automatic filling of the entire sample tube and draws up a $5-\mu l$ aliquot of blood. The cap is then placed into the body of the device, which is prefilled with the reagent solution, until passive flexural latches engage (Figure 1D). After mixing, the blood then comes into contact with the reagent solution, initiating a redox reaction (Figure 1E). Within 60 seconds, the solution undergoes a stable color change, and the Hgb level can then be estimated with the color scale sticker or with the optional smartphone app for automated analysis (Figure 1F). As normal Hgb levels range between $11.0 \mathrm{~g} / \mathrm{dl}$ and $14.5 \mathrm{~g} / \mathrm{dl}$ for children and adults $(2,17)$, the color-based POC test has been specifically optimized to detect different levels of anemia of clinical relevance: green-blue results indicate Hgb levels $<9 \mathrm{~g} / \mathrm{dl}$, representing severe anemia; yellow results indicate $\mathrm{Hgb}$ levels between 10 and $11 \mathrm{~g} / \mathrm{dl}$, representing mild anemia; and orange-red results indicate Hgb levels over $12 \mathrm{~g} / \mathrm{dl}$, representing no presence of anemia (healthy). Supplemental Video 1 depicts use of the POC ane- 
A

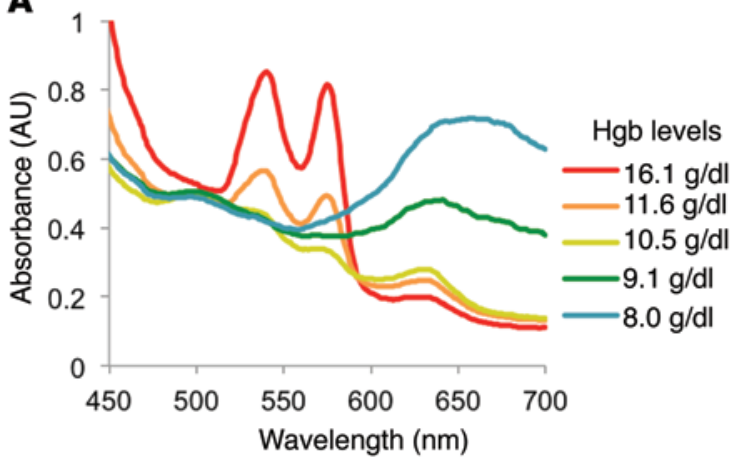

B

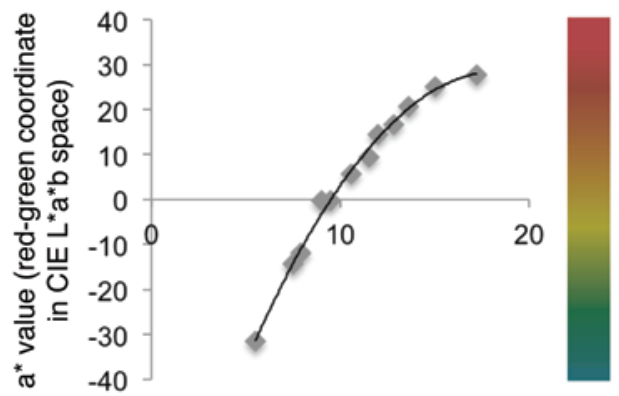

Hgb level ( $g / d l)$

Figure 2. Quantification of POC color-based anemia test results. (A) Spectrophotometric absorbance curves for resultant solution colors for blood samples with Hgb levels of $8.0 \mathrm{~g} / \mathrm{dl}, 9.1 \mathrm{~g} / \mathrm{dl}, 10.5 \mathrm{~g} / \mathrm{dl}, 11.6 \mathrm{~g} / \mathrm{dl}$, and $16.1 \mathrm{~g} / \mathrm{dl}(\lambda=450-700 \mathrm{~nm}, 5-\mathrm{nm}$ step size). Absorbance maxima for the heme peaks are at $540 \mathrm{~nm}$ and $575 \mathrm{~nm}$, and the maximum for the TMB peak is at $630 \mathrm{~nm}$. (B) Standard curve was created using a single blood sample diluted in native plasma to create Hgb levels ranging between $5.5 \mathrm{~g} / \mathrm{dl}$ and $17.2 \mathrm{~g} / \mathrm{dl}$. The final $a^{*}$ value, the coordinate in the CIE $L^{*} a^{*} b$ space representing the balance of red-green color in each resultant solution, is plotted against $\mathrm{Hgb}$ level, as measured with a standard hematology analyzer. $R$ value is 0.997 .

mia test in real time on a healthy sample (Hgb level: $15.1 \mathrm{~g} / \mathrm{dl})$, a borderline anemic sample (Hgb level: $11.5 \mathrm{~g} / \mathrm{dl}$ ), and a severely anemic sample (Hgb level: $8.0 \mathrm{~g} / \mathrm{dl}$ ).

Mechanistically, the assay involves a modification of the reaction among Hgb, hydrogen peroxide, and 3,3',5,5'-tetramethylbenzidine (TMB), in which $\mathrm{Hgb}$ catalyzes a redox reaction between TMB and hydrogen peroxide, leading to oxidized TMB products that can be detected colorimetrically (see Supplemental Figure 1 and ref. 18). This reaction has been well characterized (18-21) and has been used commercially for spectrophotometric detection of Hgb in plasma, qualitative detection of blood in stool samples, and crime scene investigation and in portable single-wavelength photometers for POC anemia testing $(22,23)$. Our group has repurposed and modified this reaction to (a) yield differential product ratios that exhibit different colorimetric properties spanning the visual spectrum, correlating with clinically relevant Hgb levels in whole blood, and (b) improve reagent stability and shelf-life. These novel modifications allow for the translation of this assay into a POC, disposable, visual color-based test for screening and monitoring anemia that does not require any additional spectrophotometric equipment or electrical power.

Standard spectrophotometrywasused to quantitativelyanalyze colorimetric aspects of our assay. Absorbance curves $(\lambda=350 \mathrm{~nm}$ to $750 \mathrm{~nm}$ ) were obtained for whole blood samples of different $\mathrm{Hgb}$ levels, ranging from $8.0 \mathrm{~g} / \mathrm{dl}$ to $16.1 \mathrm{~g} / \mathrm{dl}$ (Figure 2A). Upon completion of the redox reaction, blood samples with different $\mathrm{Hgb}$ levels yielded different resultant colors and, as expected, were associated with characteristic absorbance curves. Absorbance curves revealed different maxima for each resultant color: redcolored results (normal Hgb levels) are characterized by maxima at $540 \mathrm{~nm}$ and $575 \mathrm{~nm}$, indicating the presence of heme, whereas yellow-colored results (mild to moderate anemia) are characterized by a maximum at $450 \mathrm{~nm}$ and blue- and green-colored results (severe anemia) are characterized by a maximum at $630 \mathrm{~nm}$, indicating the presence of oxidized TMB. To enable direct quantitative comparisons with blood Hgb levels, the absorbance spectra are converted into single values, which can be accomplished by measuring the absorbance ratios at selected wavelengths or by converting the spectra into coordinates of color space systems, such as the CIE $L^{*} a^{*} b$ space $(24,25)$. Color analysis revealed that our absorbance data were primarily dependent on the red-green axis of the CIE $L^{*} a^{*} b$ space and independent of the other axes. As such, a standard curve was then created to relate Hgb levels with quantified color using $a^{*}$ values, the red-green axis CIE $L^{*} a^{*} b$ coordinates, which were converted from the absorbance data of the resultant colors of the POC anemia test (Figure 2B and ref. 23). For these experiments, blood obtained from healthy donors was diluted with native plasma to obtain samples with Hgb levels ranging from 5.5 and $17.2 \mathrm{~g} / \mathrm{dl}$, and our POC anemia test was run on each sample in quadruplicate. As shown, a second-order polynomial fit indicates a strong correlation between resultant POC test color and Hgb level ( $r=0.997)$.

A clinical assessment comparing our POC anemia test with a standard hematology analyzer was performed using blood samples obtained from patients $(n=238$; see Supplemental Table 1 for a list of patient diagnoses) with varying degrees of anemia and etiologies thereof and consisted of absorbance data, visual interpretation by untrained users, and a colorimetric smartphone app for automated analysis. As an objective measure of the accuracy of our assay, absorbance data $(\lambda=350 \mathrm{~nm}$ to $750 \mathrm{~nm})$ were obtained on a well plate reader. In addition, digital images of the resultant POC solutions were obtained via a standard smartphone camera under ambient indoor lighting conditions. To assess for assay variability, each clinical sample was tested in quadruplicate using 4 separate POC anemia tests. Supplemental Figure 2 depicts clinical patient results using our POC test $(n=238)$, as analyzed with $a^{*}$ value versus clinical hematology analyzer Hgb levels. The average of the standard deviations of the separate POC test results for each clinical sample was 1.009 in $a^{*}$ value, corresponding to $0.20 \mathrm{~g} / \mathrm{dl}$ $\mathrm{Hgb}$, establishing the inter-test variability of our POC anemia test.

Visual estimates of the POC anemia test results versus clinical hematology analyzer $\mathrm{Hgb}$ levels from patient samples $(n=238)$ is presented in Figure 3. This aspect of the clinical assessment specifically involved 12 untrained adult subjects, with no known diagnosis of color blindness, who interpreted visual data and assessed a survey of the images of POC anemia test results that were obtained for each clinical sample. This usage of images allows for the assessment of inter-user variability, as each subject will view 


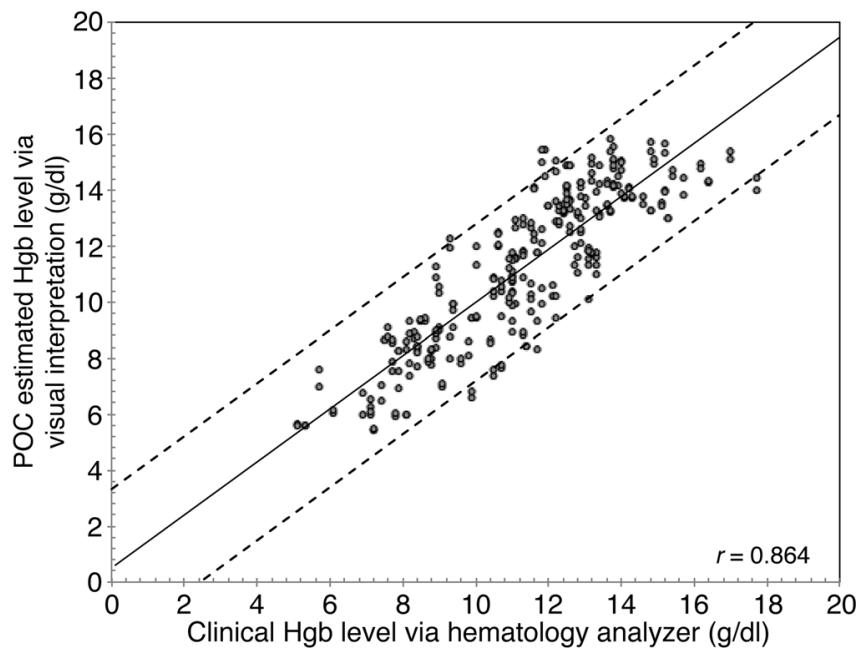

Figure 3. Clinical assessment of POC color-based anemia test results via visual interpretation. Visually interpreted Hgb level averages ( $n=12$ visual interpreters) are plotted against clinical $\mathrm{Hgb}$ levels obtained via a hematology analyzer for all patient samples $(n=238)$, showing a strong correlation $(r=0.864)$. Additionally, 95\% prediction limits for the individual values were calculated (dashed lines).

the same images, although separately and independently. Using those images, the survey subjects were then asked to estimate the Hgb levels for each clinical sample based on the set of images and corresponding Hgb levels of the standard curve POC anemia test samples (Figure 2B). Clinical Hgb levels obtained via a hematology analyzer correlated highly with visually interpreted Hgb levels $(r=0.864)$ (Figure 3). The average of the standard deviation among the surveyors' Hgb level estimates for each clinical sample was $0.67 \mathrm{~g} / \mathrm{dl} \mathrm{Hgb}$, establishing the inter-user variability of our POC anemia test. According to $\mathrm{CBC}$ results, 178 patient samples indicated presence of anemia $(<11 \mathrm{~g} / \mathrm{dl})$; of these, 10 samples indicated presence of severe anemia $(<7 \mathrm{~g} / \mathrm{dl})$. When setting anemia detection cutoffs at $7 \mathrm{~g} / \mathrm{dl}$ and at $11 \mathrm{~g} / \mathrm{dl}$, representing the detection of severe anemia and the detection of any degree of anemia, respectively, visual estimates of our POC anemia test indicate sensitivity and specificity values of $90.0 \%$ and $94.6 \%$, respectively, when detecting severe anemia $(<7 \mathrm{~g} / \mathrm{dl})$ and $90.2 \%$ and $83.7 \%$ when detecting the presence of any degree of anemia $(<11 \mathrm{~g} / \mathrm{dl})$ (Table 1$)$.

To enable automated analysis without requiring visual interpretation on the user's part as well as to enable color-blind patients to use our test, we developed an optional Java-based smartphone app that captures an image of the resultant POC anemia test and outputs an estimated Hgb level, based on CIE $L^{*} a^{*} b$ space calculations, on the screen (Figure 4A). Clinical Hgb levels obtained via a clinical hematology analyzer correlated highly with smartphone app Hgb levels $(r=0.856)$ (Figure 4B). In addition, when setting anemia detection cutoffs at $7 \mathrm{~g} / \mathrm{dl}$ and at $11 \mathrm{~g} / \mathrm{dl}$, representing the detection of severe anemia and the detection of any degree of anemia, respectively, smartphone app calculations of our POC anemia test indicate sensitivity and specificity values of $100.0 \%$ and $93.9 \%$, respectively, when detecting severe anemia $(<7 \mathrm{~g} / \mathrm{dl})$ and $91.1 \%$ and $79.2 \%$ when detecting the presence of any degree of anemia $(<11 \mathrm{~g} / \mathrm{dl})$ (Table 2$)$. Clinical adult and pediatric visual interpretation and smartphone app analysis data are presented separately in Supplemental Figure 3.
As a potential home diagnostic test, we also tested the capability of our POC anemia test for serial Hgb level monitoring. Figure 5 depicts POC color-based results over 45 days for a healthy control male, a healthy control female, and a male with $\beta$-thalassemia major, a genetic disease that causes chronic anemia, who receives monthly red blood cell transfusions. Absorbance ratios (540 nm/630 nm) were used to estimate Hgb levels, which were in agreement with the subjects' corresponding Hgb levels obtained via hematology analyzer obtained the same days as the POC anemia tests were performed (Figure 5). As shown, the Hgb levels of the control male do not show any significant deviation from baseline throughout the course of the study. In contrast, the Hgb levels of the female control indicate lower Hgb at days 15 and 41, which coincided with menstruation. The Hgb levels of the thalassemic subject were low on days 3,27 , and 41 , which coincided with the days just prior to red cell transfusion. Overall, the color-based POC test was able to accurately track distinguishable Hgb level changes over time.

\section{Discussion}

This color-based POC anemia test offers rapid, simple, inexpensive, and disposable self-screening and self-monitoring for anemia at home, requiring only a finger stick drop of capillary blood. The key unique aspects are its (a) visual color-based readout, enabling its use as a stand-alone system that does not require electrical power or additional equipment (such as a single-wavelength photometer); (b) low cost (each device currently costs US $\$ 0.50$ to manufacture and is disposable); (c) small size and portability; and (d) ease of use, which enables users and patients to test themselves at their convenience. We have also developed an optional colorimetric smartphone app, with which the user simply takes an image of the resultant color change of the POC anemia test and the app outputs an estimated $\mathrm{Hgb}$ level on the smartphone screen. While not a requirement, this app provides automated analysis, with the built-in color scale sticker for users who are color-blind or prefer to not use their own judgment in estimating Hgb levels.

A clinical assessment determined that the POC anemia test obtained similar results to those of hematology analyzers $(r=0.864$ and $r=0.856$ for visual interpretation and smartphone app, respectively), and both POC test analysis methods yielded comparable sensitivity and specificity for detecting anemia $(<11 \mathrm{~g} / \mathrm{dl})$ (sensitivity: $90.2 \%$ and $91.1 \%$, specificity: $83.7 \%$ and $79.2 \%$, respectively) and severe anemia ( $<7 \mathrm{~g} / \mathrm{dl})$ (sensitivity: $90.0 \%$ and 100\%, specificity: $94.6 \%$ and $93.9 \%$, respectively). These values are similar to those of existing POC anemia diagnostic tests, which currently are not designed for, and are not practical for, home use or use as a self-test $(12-17,26,27)$. These data also highlight the

Table 1. Sensitivity and specificity via visual interpretation

$\begin{array}{lcc} & \text { Sensitivity } & \text { Specificity } \\ \text { Severe anemia }(<7 \mathrm{~g} / \mathrm{dl}) & 90.0 \% & 94.6 \% \\ \text { Mild anemia }(<11 \mathrm{~g} / \mathrm{dl}) & 90.2 \% & 83.7 \%\end{array}$

Sensitivity and specificity of POC estimated $\mathrm{Hgb}$ via visual interpretation were calculated for detecting severe anemia $(<7 \mathrm{~g} / \mathrm{dl})$ and the presence of any anemia $(<11 \mathrm{~g} / \mathrm{dl})$. 
A
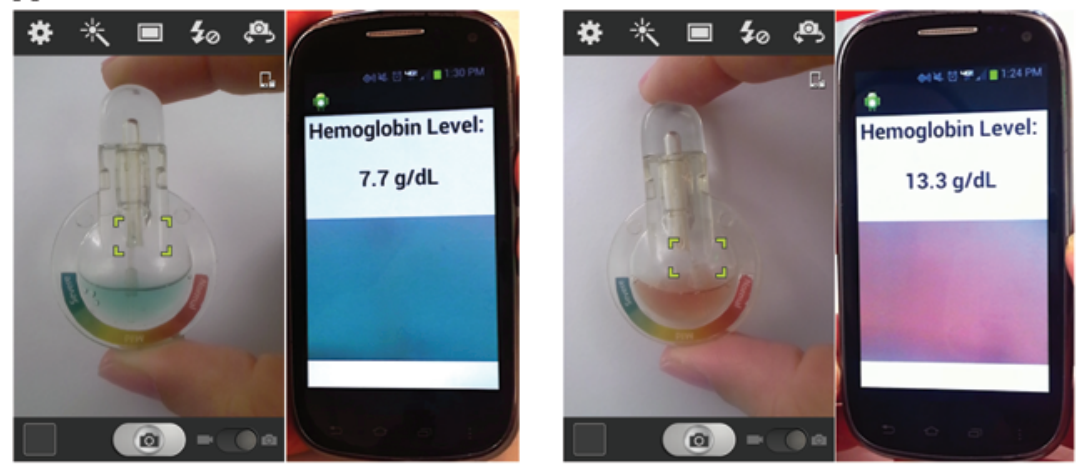

B

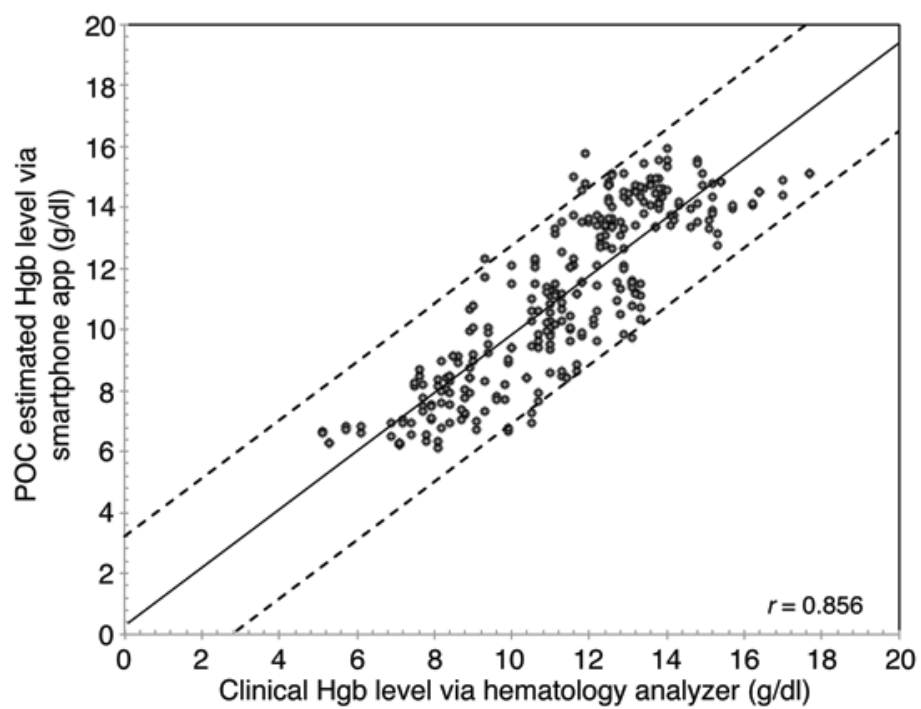

Figure 4. Clinical assessment of POC color-based anemia test results via optional smartphone app. (A) Pictures depict the Java-based smartphone app analyzing anemic and healthy samples. The smartphone app obtains an image of the resultant solution color for an anemic sample $(8 \mathrm{~g} / \mathrm{dl})$ and for a healthy sample $(13 \mathrm{~g} / \mathrm{dl})$ and outputs Hgb levels of $7.7 \mathrm{~g} / \mathrm{dl}$ and $13.3 \mathrm{~g} / \mathrm{dl}$ for the anemic and healthy samples, respectively, on the screen. (B) Smartphone app $\mathrm{Hgb}$ levels are plotted against clinical Hgb levels obtained via a hematology analyzer for all patient samples $(n=238)$, showing a strong correlation $(r=0.856)$. Additionally, 95\% prediction limits for the individual values were calculated (dashed lines).

clinical utility of the smartphone app. While optional, as the POC anemia test is functional as a stand-alone device, the smartphone app not only enables automated analysis, color-blind users to operate the system, and the possibility of remote data transmission, it also increases the sensitivity of the test. Our continued development of the smartphone app will therefore likely further increase sensitivity of the POC anemia test.

Even in developed nations, this POC anemia test would serve multiple demographics and patient populations. For example, because of its low cost and ease of use, this system could be a cost-effective and convenient anemia self-screening method for the general population, especially those at risk for anemia, including pregnant women, young children, and the elderly. In addition, chronically anemic patients, such as cancer patients receiving chemotherapy, patients with renal failure, patients with chronic inflammatory/immunologic disorders, or patients with primary hematologic disorders (e.g., sickle cell anemia, thalassemia, aplastic anemia), could use this POC anemia test to self-monitor their anemia and seek medical attention when their $\mathrm{Hgb}$ levels decrease significantly from their baseline $(9-11,28)$. Indeed, chronic hemolytic anemia patients could potentially use this system for early self-detection of worsening anemia, which could be sudden, rapid, and potentially life threatening, in the context of viral infections or onset of acute complications in the case of sickle cell disease Finally, as our POC anemia test requires a small amount of blood ( $5 \mu \mathrm{l})$ from a simple finger stick, this diagnostic would be especially useful to minimize blood loss due to laboratory testing in neonates and children, especially if Hgb levels need to be checked repeatedly (26).

In addition, our current chemical formulation yields color results correlating to different levels of anemia, as defined by the World Health Organization, $<7 \mathrm{~g} / \mathrm{dl}$ for severe anemia and $<11 \mathrm{~g} / \mathrm{dl}$ for mild anemia (29). This chemical formulation can be altered in a finely tuned manner to "shift" the color scale to correspond to different ranges of Hgb levels (Supplemental Figure 4). As shown, the resultant solution colors of the current chemical formulation accurately differentiate between Hgb levels of $5.5 \mathrm{~g} / \mathrm{dl}$ and $17.2 \mathrm{~g} / \mathrm{dl}$. The leftshifted curve depicts another formulation of the POC anemia test that can produce different color results corresponding to $\mathrm{Hgb}$ levels ranging from $2.5 \mathrm{~g} / \mathrm{dl}$ to $9.1 \mathrm{~g} / \mathrm{dl}$ and would be especially useful for diagnosing patients with severe anemia in the global health and low-resource settings. In contrast, the right-shifted curve depicts another formulation designed to detect and differentiate $\mathrm{Hgb}$ levels ranging from $13.6 \mathrm{~g} / \mathrm{dl}$ to $25.4 \mathrm{~g} / \mathrm{dl}$, and, as such, this formulation can be used to accurately diagnose the mildest forms of anemia as well as differing degrees of polycythemia. Therefore, the exact formulation of the POC anemia test will be dictated by the disease type and clinical scenario.

While several devices are currently available for POC anemia testing, the vast majority of these systems remain in the clinical setting, due to high costs and required technical skill for use, in comparison with our POC test. For example, portable POC devices that measure Hgb levels exist, but they are not stand-alone, consisting of an electrically powered analyzer and disposable cartridges, and also require technical training $(13,26)$. The majority of these analyzers cost between US $\$ 1,000$ and $\$ 2,000$, with each test itself costing US \$1-2 thereafter. More recently, noninvasive co-oximeters offering continuous Hgb level measurements have been developed, but they cost between US $\$ 1,000$ and $\$ 3,000$ and also require electrical power and technical training $(17,22)$. In an attempt to introduce a simple, low-cost alternative for low-resource settings, the World Health Organization developed the Haemoglobin Colour Scale to test for anemia using the color of one's blood to differentiate between Hgb levels, such that anemic samples reflected a paler red color as compared with that of normal samples (12-16). While initial results indicated acceptable 
Table 2. Sensitivity and specificity via smartphone app

$\begin{array}{lcc} & \text { Sensitivity } & \text { Specificity } \\ \text { Severe anemia }(<7 \mathrm{~g} / \mathrm{dl}) & 100 \% & 93.9 \% \\ \text { Mild anemia }(<11 \mathrm{~g} / \mathrm{dl}) & 91.1 \% & 79.2 \%\end{array}$

Sensitivity and specificity of POC estimated $\mathrm{Hgb}$ via smartphone app were calculated for detecting severe anemia $(<7 \mathrm{~g} / \mathrm{dl})$ and the presence of any anemia $(<11 \mathrm{~g} / \mathrm{dl})$.

accuracy, follow-up field studies revealed suboptimal sensitivity and specificity, likely due to the level of technical training required to accurately perform and interpret the test (12).

As with any biochemical assay, our modified TMB-mediated reaction could be confounded by potential interfering substances. For blood, in particular, potential interference from byproducts of red cell destruction, which occur in hemolytic anemias (e.g., sickle cell anemia, thalassemias) must be assessed. Within our clinical results, we determined that reticulocyte count, a metric of the degree of hemolysis, did not correlate with POC Hgb level deviation from the clinical Hgb level obtained from hematology analyzers. In addition, to determine whether the presence of bilirubin adversely affected the results of our POC anemia test, we systematically added bilirubin to existing blood samples, conducted our assay, and measured our assay's Hgb levels based on spectrophotometry absorbance data. As shown in Supplemental Figure 5A, bilirubin levels between 0 and $5 \mathrm{mg} / \mathrm{dl}$ have negligible effects on our POC anemia test, and significant effects only occur at nonphysiologic levels above $10 \mathrm{mg} / \mathrm{dl}$. In addition, we determined that the presence of lipids in blood samples did not affect test results and neither did varying blood $\mathrm{pH}$ within physiological levels (Supplemental Figure 5, B and C). Elevated levels of myoglobin can be detected in the blood following trauma (30), but even the highest tested myoglobin concentrations $(25 \mu \mathrm{l} / \mathrm{mg})$ did not affect the POC anemia test results (Supplemental Figure 5D). The required redox reaction is affected by temperature and light, so each device is stored at $24^{\circ} \mathrm{C}$ in the dark until just prior to use. In addition, the devices can be stored at between $0^{\circ} \mathrm{C}$ and $40^{\circ} \mathrm{C}$ and remain accurate when equilibrated to and tested at room temperature $\left(20^{\circ} \mathrm{C}-28^{\circ} \mathrm{C}\right)$. The resultant colors are stable for over 15 minutes after the reaction is complete, and shelf life studies on our POC test revealed that our premixed solutions stored at $24^{\circ} \mathrm{C}$ in the dark remained accurate after 6 months.

In today's healthcare landscape, containing costs while improving patient empowerment and quality of life is of extreme importance. As such, POC diagnostics are taking on larger and more important roles in patient care; interest in developing homebased self-tests, as ubiquitous as urine pregnancy tests and diabetic glucometers, is only increasing. Anemia is one of the most commonly diagnosed medical conditions, and the color-based POC test presented here offers a simple, rapid, and inexpensive way to self-test for anemia with a single drop of blood from a simple finger stick. In addition, we also envision our POC anemia test will be especially useful in low-resource settings, in which hematology analyzers are cost prohibitive; studies are ongoing. Finally, the small blood volumes required of this assay enable it to be an ideal POC anemia test for neonates and pediatric patients. Over- all, our results demonstrate the feasibility of this POC anemia test to function as a self-screening and self-monitoring tool for any patient who has or is at risk for anemia.

\section{Methods}

Color-based POC anemia testing. Each color-based POC anemia device was prefilled with $500 \mu \mathrm{l}$ of a modified TMB and reagent solution and was coupled with a 5 - $\mu$ l straight, sodium heparin-coated, capillary tube (Innovative Med Tech) for blood collection. Blood sample preparation for standard calibration curves experiments (Figure 2) and stability testing involved obtaining blood from a healthy subject via venipuncture procedure into a heparinized container. Blood was then centrifuged at $150 \mathrm{~g}$ for 10 minutes to separate plasma from red blood cells. Different dilutions (ranging from $6.8 \mathrm{~g} / \mathrm{dl}$ to $17 \mathrm{~g} / \mathrm{dl}$, as indicated) were prepared by mixing different ratios of native red blood cells and platelet poor plasma. Hgb levels were determined via an Advia 2120 Hematology System. All device prototypes were fabricated in the Global Center for Medicine Innovation.

Obtaining and processing patient blood samples. All patients enrolled in our study obtained a CBC for their clinical care the same day, typically immediately before, blood was obtained for the color-based POC anemia test. Patient samples were obtained, and CBCs, which include a Hgb level, were run on standard clinical grade hematology analyzers (Advia 2120i, Siemens Inc. and Heska Hematrue, Heska). Patient blood samples for the color-based POC anemia test were collected via finger stick with a spring-loaded lancing device (One Touch Ultra Soft) and a disposable 21-gauge lancet. As quadruplicate measurements using the color-based POC anemia test were conducted for each sample $(n=4), 100-200 \mu \mathrm{l}$ capillary blood was collected from each patient subject. Once the assay reaction was complete, equal portions of the solution were transferred into 4 wells of a 96-well plate. Absorbance values across the visual spectrum ( $\lambda=350 \mathrm{~nm}$ to $750 \mathrm{~nm}, 5-\mathrm{nm}$ step size) were then recorded using a well plate spectrophotometer (SpectraMax M2/M2e).

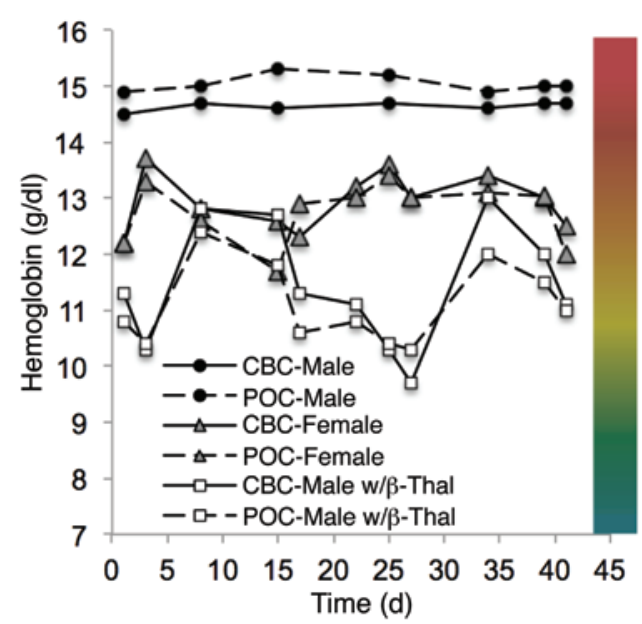

Figure 5. Serial Hgb level monitoring with the POC anemia test. Over 45 days, blood samples were obtained serially from a healthy adult man, a healthy adult woman, and a man with $\beta$-thalassemia major and tested via CBCs using a standard hematology analyzer and POC anemia test. Days 11 and 37 mark the first day of the female subject's menstrual cycle, which is reflected with lower $\mathrm{Hgb}$ levels on days 15 and 41. The man with $\beta$-thalassemia major was transfused on days 7 and 30 , indicating relatively higher Hgb levels than his anemic baseline. 
Overall, 238 clinical blood samples from pediatric and adult patients were collected, tested, and analyzed using our POC anemia test and were compared with the patients' clinical CBC results. There were no adverse effects experienced by study subjects. In addition, one healthy adult male, one healthy adult female, and one male with $\beta$-thalassemia major were recruited for serial testing over 45 days ( $n=1$ data point per subject per day tested). For this serial study, each sample was tested using our POC anemia test and a hematology analyzer, either an Advia 2120 Hematology System or a Heska Hematrue analyzer (shown to correlate with the Advia system, $r=0.998$ ). The research technician was blinded to golden standard $\mathrm{CBC}$ results until testing was complete. Each sample was tested in quadruplicate $(n=4)$ using the POC anemia test.

Assay color quantification via $a^{*}$ value analysis. To enable head-tohead comparison of the color-based POC anemia test with Hgb levels obtained from a standard clinical hematology analyzer, the color changes the reagent undergoes must be quantified and distilled to a single number. One method commonly used in spectroscopy is to take the ratio of 2 absorbance intensity peaks, as shown in Figure 2A. Another method involves conversion of absorbance data into CIE $L^{*} a^{*} b^{*}$ space data (24). Absorbance data across the visual spectrum ( $\lambda=350 \mathrm{~nm}$ to $750 \mathrm{~nm}, 5-\mathrm{nm}$ step size) were obtained with a well plate spectrophotometer (SpectraMax M2/M2e) and imported into MATLAB. The code analyzed color as a value of $a^{*}$ and correlated values to Hgb levels using the procedure described below.

Colors correspond to the CIE $L^{*} a^{*} b^{*}$ (CIELAB) color space. The CIELAB space is a tricoordinate color space, wherein the $L^{*}, a^{*}$, and $b^{*}$ coordinates correspond to the white-black, red-green, and yellow-blue balances of a given color, respectively $(24,25)$. The CIELAB color space was originally generated to allow for a perceptually uniform color space, hence changes in colors should accompany a change in quantified color value (24). To generate comparable color data in the CIELAB space, absorbance data spanning $\lambda=350 \mathrm{~nm}$ to $750 \mathrm{~nm}, 5-\mathrm{nm}$ step size, was used to first calculate color values in the CIE $1931 \mathrm{XYZ}$ space, using the equations listed below.

$$
X=K \int_{350}^{750} S(\lambda) \bar{x}(\lambda) R(\lambda) d(\lambda)
$$

(Equation 1)

$$
Y=K \int_{350}^{750} S(\lambda) \bar{y}(\lambda) R(\lambda) d(\lambda)
$$

(Equation 2)

$$
Z=K \int_{350}^{750} S(\lambda) \bar{Z}(\lambda) R(\lambda) d(\lambda)
$$

$$
\text { (Equation 3) }
$$

$$
K=\frac{100}{\int_{350}^{750} S(\lambda) \bar{y}(\lambda) d(\lambda)}
$$

(Equation 4)

$S(\lambda)$ is the relative spectral power distribution of a standard illuminant, in this case a D50 source. $\bar{x}(\lambda), \bar{y}(\lambda)$, and $\bar{z}(\lambda)$, supplied by the CIE, are the 1931 spectral tristimulus values. $R(\lambda)$ is the spectral reflectance (or transmittance) of the object measured. The values of $X, Y$, and $Z$ were then used to calculate values for $L^{*}, a^{*}$, and $b^{*}$ using the following equations:

$$
\begin{aligned}
& L^{*}=116\left(\frac{Y}{Y_{n}}\right)^{\frac{1}{3}}-16 \\
& a^{*}=500\left[\left(\frac{X}{X_{n}}\right)^{\frac{1}{3}}-\left(\frac{Y}{Y_{n}}\right)^{\frac{1}{3}}\right] \\
& b^{*}=200\left[\left(\frac{Y}{Y_{n}}\right)^{\frac{1}{3}}-\left(\frac{Z}{Z_{n}}\right)^{\frac{1}{3}}\right]
\end{aligned}
$$

(Equation 5)

(Equation 6)

(Equation 7)

$X_{n}, Y_{n}$, and $Z_{n}$ are the CIE tristimulus of the reference white point. A script to process these calculations was written in MATLAB. It was noted that the $L^{*}$ and $b^{*}$ values were largely independent of the Hgb levels. As such, $a^{*}$ values, which are dependent on the red-green color balance in each result, correlate to our color scale and, therefore, the Hgb level for each sample (Figure 2 and Supplemental Figure 2).

Assay color quantification via an optional smartphone app. Colorimetric analysis was performed using custom software written in MATLAB. A calibration function relating Hgb concentrations to peak histogram values for each RGB channel was used to estimate the Hgb concentration for all sample images. The calibration function was created using images of processed POC anemia devices containing blood samples with known Hgb levels (the same standard calibration results presented in Figure 2B). The center 25\% of each calibration image was selected as the region of interest (ROI). RGB values in the ROI were converted to CIE $L^{*} a^{*} b^{*}$ color space using a D50 luminator white point. A histogram for each channel $\left(L^{*}, a^{*}\right.$, and $\left.b^{*}\right)$ in the ROI was calculated. Peaks of the channel histograms for each calibration image and corresponding Hgb value were fit using planar regression with the following function: estimated Hgb level $=\mathfrak{w}+x^{*}\left(L_{\text {peak }}^{*}\right)+y^{*}\left(a_{\text {peak }}^{*}\right)+z^{*}\left(b_{\text {peak }}^{*}\right)$.

This MATLAB function was then converted into a Java-based app for performance on an Android-powered mobile phone. The smartphone app allows the user to take a digital photographic image of the resultant solution color and obtain an estimated digital Hgb level result based on the same function used in MATLAB. After image capture, the app saves the image and outputs an estimated Hgb level on screen (Figure 4A). Using this app, Hgb concentrations were estimated for clinical samples (Figure 4B).

Visual-based analysis. Using a standard smartphone (iPhone 4, iOS 6) set to default lighting and focusing, images were obtained of all processed POC anemia tests (the entire device, including the reagent solutions after the reaction was completed) using patient blood samples. These images were then were placed in randomized order and compiled into surveys. A blinded study was then conducted in which 12 untrained adult subjects (6 women and 6 men with no known diagnosis of any form of color blindness) were asked to visually interpret and assign $\mathrm{Hgb}$ values of the images using a color scale, which was based on colors obtained from POC anemia tests of different but known Hgb levels.

Statistics. All statistical analyses were performed using SAS 9.3. A $P$ value of less than 0.05 was considered significant. Pearson correlations and general linear regression models and prediction limits were used to assess associations between Hgb level via hematology analyzer and absorbance ratio, $a^{*}$ value, visually interpreted Hgb level, and smartphone app Hgb level. Individual patients were identified 
as either adult or pediatric and investigated for differences in their outcomes via analysis of covariance, controlling for the Hgb level via hematology analyzer covariate. In cases when the assumption of equal regression slopes was violated, linear regression models were run separately for adult and pediatric patients. Sensitivity and specificity results were calculated with both adult and pediatric patients together as well as by considering each patient type individually.

Study approval. Pediatric and adult patient blood samples were obtained in outpatient clinics of the Aflac Cancer and Blood Disorders Center of Children's Healthcare of Atlanta and Emory's Winship Cancer Center, respectively, by one consistent research technician using protocols approved by the IRBs of Emory University, Georgia Institute of Technology, and Children's Healthcare of Atlanta. All samples were acquired after informed consent was obtained from patients or their parents/guardians. Each consented patient only provided one sample on the day of consent.

\section{Acknowledgments}

We thank the following funding sources: the FDA-funded Atlantic Pediatric Device Consortium (P50FD004193), the Georgia
Research Alliance for phase 1 venture funding, the Children's Healthcare of Atlanta-sponsored summer internship program, the Georgia Center of Innovation for Manufacturing, and the InVenture Prize and the Ideas to Serve competitions at the Georgia Institute of Technology. We thank the physicians and nurse practitioners of the Aflac Cancer and Blood Disorders Center at Children's Healthcare of Atlanta and the physicians and nurse practitioners of the Winship Cancer Institute at Emory University who assisted with the collection of clinical samples. We also thank the Global Center for Medical Innovation (GCMI) for all prototype iteration and production. We thank M. Byrd, N. Hotaling, M. McKinnon, and M. Platt for initial discussions, advice, and help with initial experiments. We also thank C.R. Fantz for assistance in POC testing and stability assessment. We finally thank the entirety of the Lam lab, including Y. Sakurai, E.T. Hardy, D.R. Myers, H.A. Gole, Y. Qiu, B. Ahn, R. Tran, J. Ciciliano, and M. Fay for thoughtful discussions.

Address correspondence to: Wilbur A. Lam, 2015 Uppergate Drive, Emory Children's Center - Room 448, Atlanta, Georgia 30332, USA. Phone: 404.727.7473; E-mail: wilbur.lam@emory.edu.
1. World Health Organization. Micronutrient deficiencies. WHO Web site. http://www.who.int/ nutrition/topics/ida/en/. Accessed July 30, 2014.

2. Petranovic D, Batinac T, Petranovic D, Ruzic A, Ruzic T. Iron deficiency anaemia influences cognitive functions. Med Hypotheses. 2008;70(1):70-72.

3. Carter RC, et al. Iron deficiency anemia and cognitive function in infancy. Pediatrics. 2010;126(2):e427-e434.

4. Calabrich A, Katz A. Management of anemia in cancer patients. Future Oncol. 2011;7(4):507-517.

5. Beghe C, Wilson A, Ershler WB. Prevalence and outcomes of anemia in geriatrics: a systematic review of the literature. Am JMed. 2004;116(suppl 7A):3S-10S.

6. Koukounari A, et al. Relationships between anaemia and parasitic infections in Kenyan schoolchildren: a Bayesian hierarchical modelling approach. Int J Parasitol. 2008;38(14):1663-1671.

7. Bechir M, Schelling E, Hamit MA, Tanner M, Zinsstag J. Parasitic infections, anemia and malnutrition among rural settled and mobile pastoralist mothers and their children in Chad. Ecohealth. 2012;9(2):122-131.

8. Meidani M, Rezaei F, Maracy MR, Avijgan M, Tayeri K. Prevalence, severity, and related factors of anemia in HIV/AIDS patients. JRes Med Sci. 2012;17(2):138-142.

9. Leenstra T, et al. Schistosomiasis japonica, anemia, and iron status in children, adolescents, and young adults in Leyte, Philippines 1. Am J Clin Nutr. 2006;83(2):371-379.

10. Friedman JF, Kanzaria HK, McGarvey ST. Human schistosomiasis and anemia: the relationship and potential mechanisms. Trends Parasitol. 2005;21(8):386-392.

11. Isanaka $S$, et al. Iron deficiency and anemia predict mortality in patients with tuberculosis. JNutr. 2012;142(2):350-357.
12. Critchley J, Bates I. Haemoglobin colour scale for anaemia diagnosis where there is no laboratory: a systematic review. Int J Epidemiol. 2005;34(6):1425-1434.

13. Paddle JJ. Evaluation of the Haemoglobin Colour Scale and comparison with the HemoCue haemoglobin assay. Bull World Health Organ. 2002;80(10):813-816.

14. White SA, van den Broek NR. Methods for assessing reliability and validity for a measurement tool: a case study and critique using the WHO haemoglobin colour scale. Stat Med. 2004;23(10):1603-1619.

15. Ingram CF, Lewis SM. Clinical use of WHO haemoglobin colour scale: validation and critique. JClin Pathol. 2000;53(12):933-937.

16. Aldridge C, Foster HM, Albonico M, Ame SM, Montresor A. Evaluation of the diagnostic accuracy of the Haemoglobin Colour Scale to detect anaemia in young children attending primary healthcare clinics in Zanzibar. Trop Med Int Health. 2012;17(4):423-429.

17. Dewhirst E, et al. Accuracy of noninvasive continuous hemoglobin measurement by pulse co-oximetry during preoperative phlebotomy. J Intensive Care Med. 2013;29(4):238-242.

18. Josephy PD, Eling T, Mason RP. The horseradish peroxidase-catalyzed oxidation of 3,5,3',5'-tetramethylbenzidine. Free radical and charge-transfer complex intermediates. J Biol Chem. 1982;257(7):3669-3675.

19. Reynolds M, Lawlor E, McCann SR, Temperley IJ. Use of 3,3',5,5'-tetramethylbenzidine (TMB) in the identification of erythroid colonies. J Clin Pathol. 1981;34(4):448-449.

20. Levinson SS, Goldman J. Measuring hemoglobin in plasma by reaction with tetramethylbenzidine. Clin Chem. 1982;28(3):471-474.

21. Zierdt WS, Zierdt CH. Occult blood testing using tetramethylbenzidine in an extraction procedure for patients on unrestricted diets. Am JClin Pathol. 1985;83(4):486-488.

22. McMurdy JW, Jay GD, Suner S, Crawford G. Noninvasive optical, electrical, and acoustic methods of total hemoglobin determination. Clin Chem. 2008;54(2):264-272.

23. Laberke PJ, Hausmann R, Wiprachtiger N, Briellmann T, Balitzki B. [Analysis with the Combur-Test - special aspects in forensic trace examination]. Arch Kriminol. 2012; 229(5-6):189-197.

24. Mortimer RJ, Varley TS. Quantification of colour stimuli through the calculation of CIE chromaticity coordinates and luminance data for application to in situ colorimetry studies of electrochromic materials. Displays. 2011;32(1):35-44.

25. Guild J. The colorimetric properties of the spectrum. Philos T R Soc Lond. 1932;230A:149-187.

26. Lamhaut L, Apriotesei R, Combes X, Lejay M, Carli P, Vivien B. Comparison of the accuracy of noninvasive hemoglobin monitoring by spectrophotometry ( $\mathrm{SpHb}$ ) and HemoCue(R) with automated laboratory hemoglobin measurement. Anesthesiology. 2011;115(3):548-554.

27. Nkrumah B, et al. Hemoglobin estimation by the HemoCue(R) portable hemoglobin photometer in a resource poor setting. BMC Clin Pathol. 2011;11:5.

28. Kristinsson G, Shtivelman S, Hom J, Tunik MG. Prevalence of occult anemia in an urban pediatric emergency department: what is our response? Pediatr Emerg Care. 2012;28(4):313-315.

29. World Health Organization. Worldwide prevalence of anaemia 1993-2005. WHO Global Database on Anaemia. http://whqlibdoc.who.int/publications/2008/9789241596657_eng.pdf. Atlanta, Georgia, USA: Center for Disease Control; 2005.

30. Olerud JE, Homer LD, Carroll HW. Incidence of acute exertional rhabdomyolysis. Serum myoglobin and enzyme levels as indicators of muscle injury. Arch Intern Med. 1976;136(6):692-697. 